\title{
The Influence of Partisan Media in the Face of Global Pandemic: How News Shaped COVID-19 Vaccine Hesitancy
}

\author{
Matt Motta, PhD \\ Assistant Professor \\ Department of Political Science \\ Oklahoma State University \\ matthew.motta@okstate.edu
}

\author{
Dominik Stecula, PhD \\ Assistant Professor \\ Department of Political Science \\ Colorado State University \\ dominik.stecula@colostate.edu
}

If any event might interrupt conventional partisan media effect processes, some might expect the promise of ending a global pandemic via vaccination to do so. We test that possibility by bringing together sentiment-scored COVID vaccine stories $(N>17,000)$ from cable and mainstream news outlets, $N>180,000$ government-registered adverse event reports, and six original surveys $(N=6,499)$, in order to investigate (1) whether partisan news outlets covered COVID vaccination in different ways, and (2) if differences in coverage increased vaccine hesitancy. We find that Fox News' (FXNWS) coverage was significantly more negative than that of other cable and mainstream sources. Worryingly, FXNWS vaccine coverage increased public vaccine hesitancy. In the aggregate, adverse event reports increased following periods of heightened negativity on FXNWS. At the micro-level, self-reported FXNWS exposure is associated with increased vaccine refusal. Collectively, the results provide new insights into the persuasive power of partisan media.

Keywords: Media Effects, Partisan Media, COVID-19, Health Politics, Public Opinion Word Count: 7,643 
As of this writing, the death toll of the global COVID-19 pandemic has been over 5.6 million, with nearly 900 thousand of these deaths happening in the United States. ${ }^{1}$ The pandemic has directly affected the lives of every American in one way or another; via job loss, interruption in their children's schooling, caring for sick loved ones, and/or even contracting COVID themselves. Because the pandemic has had a profoundly personal effect on the lives of many (if not most) Americans, is precisely the kind of event where we would expect the content of the news media to matter less than direct, lived experience.

Traditionally, the influence of the news media is the strongest on issues with which people do not necessarily have direct experience (Lecheler et al., 2009; Yagade \& Dozier, 1990; McCombs, 2005; Shafi, 2017). Whether it's the state of the national economy, withdrawing armed forces from Afghanistan, or Congressional negotiations over the infrastructure bill, most Americans do not have direct, personal experience with these issues, and rely on the portrayal in the news media to shape their beliefs and attitudes. But with COVID, they actually experience the pandemic first hand themselves, potentially making mediated COVID coverage less important.

At the same time, the politicization of COVID - and the issue of vaccination in particular, which public health officials cite as essential for putting the pandemic's spread into declinehas been extraordinary, and the pandemic clearly became a tremendously salient culture war issue (Pickup et al., 2020; Gadarian et al., 2021; Clinton et al., 2021; Grossman et al., 2020; Allcott et al., 2020). In general, Democratic elites embraced COVID positions promoted by health experts like Dr. Anthony Fauci on masking, social distancing, and vaccines. Republican

\footnotetext{
${ }^{1}$ https://covid19.who.int
} 
elites, especially those aligned with the world of Donald Trump and conservative talk radio, on the other hand, took a stance in opposition to Democrats, which includes discourse that is hostile of masking and skeptical of vaccines. Correspondingly, we might expect loyal partisans to put aside their personal experiences with the virus and embrace elite talking points mediated through partisan media outlets, especially since these sources are much more likely to include the most extreme voices and the most outlandish and misinforming claims (Motta et al., 2020).

In this paper, we offer a novel and methodologically pluralistic series of tests devised to assess whether or not partisan news media play a role in shaping attitudes about COVID vaccines. We first examine how the national news media, including major national newspapers and cable news outlets, covered COVID-vaccination in the early months of the vaccine rollout. We pay particular attention to Fox News, given its importance as a trusted news source among Republicans, the lack of trust among Republicans in other news sources, and the network's track record in spreading COVID and vaccine misinformation. We then look at how the news coverage of COVID vaccines influenced public vaccine hesitancy, using both self-reported (public opinion) and quasi-behavioral data; the latter of which is measured through the vaccine adverse event reports to the Department of Health and Human Services.

We find, via automated sentiment analyses, that Fox News covered the vaccines in much more negative light than other news outlets. Differences in media coverage had a profound impact on public opinion, which we assess in two ways. First, we present time-varying evidence adverse event reports (a proxy for public vaccine sentiment; see Motta \& Stecula 2021) tend to increase following heightened periods of negativity on Fox News (but not on 
other outlets). Second, survey evidence fortifies these claims by showing that Fox News viewers reported elevated levels of vaccine hesitancy throughout the pandemic.

These results showcase the persuasive power of partisan media. While some might expect the promise of vaccination to put an end to a global pandemic, and Americans' highlypersonal experiences with it, to interrupt conventional media effect processes, we find that differences in covered vaccine-related issues had both predictable and polarizing effects on public opinion.

\section{Media Effects during a Global Pandemic}

Decades of work in the domain of political communication has demonstrated the power of the news media to shape attitudes and behaviors across various political domains. The news media chooses which issues to cover and how frequently, and hence influences what issues the public deems important (McCombs \& Shaw, 1972). The nature of coverage, how issues are framed, and what considerations are given increased attention (potentially at the exclusion of others) are also important in shaping public attitudes about an issue (Scheufele \& Tewksbury, 2007). Of course, the media will not shape attitudes on all topics equally. Political phenomena with which Americans have highly direct and personal experience will be less susceptible to media effects than more complex issues, such as climate change (Merkley \& Stecula, 2021).

Taken together, this suggests that during an event like the global pandemic, the influence of the news media should have less capacity to persuade than when it comes issues with which the public has less direct experience. That is not to say that the media would not necessarily shape opinion during a public health crisis, given the extraordinary amount of 
attention that Americans have been paying to COVID-related news. ${ }^{2}$ Previous work has shown that increased media exposure to a public health crisis influences not only issue salience but also health behavior intentions (Haglin et al., 2019). Furthermore, given the disruptions to Americans' daily lives, and the fact that COVID-vaccines are essential for putting the virus' spread into decline ${ }^{3}$, the expectation should be that media effects on the topic of vaccination specifically should be subdued. The media might influence opinion when it comes to issue positions on distal, complex, policy problems. But, when it comes to life and death COVID decisions and the desire to return to normal, past research leads us to suspect that the ability of the news media to shape public opinion should be less pronounced (Lecheler et al., 2009).

\section{Politicization of COVID}

Interestingly, the COVID pandemic have showcased that insights from previous research may need to be revisited. Unlike in other western democracies, the COVID pandemic has been politicized in the United States to an exceptional degree (Merkley et al., 2020; Sol Hart et al., 2020; Pickup et al., 2021). Almost from the very beginning of the pandemic, political elites talked about COVID differently and polarized on this issue along party lines (Green et al., 2020). In general, Republicans, led by President Donald Trump, dismissed the seriousness of the disease, downplayed the need for public health mitigation measured like wearing masks or social distancing, which was in stark opposition to what Democrats and the scientific community were promoting. As a result, the public has polarized on this issue relatively quickly, with stark differences across party lines on not only COVID attitudes and beliefs, but also

\footnotetext{
${ }^{2}$ https://www.pewresearch.org/journalism/2020/03/18/americans-immersed-in-covid-19-news-most-think-media-are-doingfairly-well-covering-it/

${ }^{3}$ https://www.reuters.com/world/us/fauci-says-he-expects-more-covid-vaccine-approvals-coming-weeks-2021-08-24/
} 
behaviors (Pickup et al., 2020; Gadarian et al., 2021; Clinton et al., 2021; Grossman et al., 2020; Allcott et al., 2020).

Whether we should expect similar pattern of polarization on COVID vaccinations is a separate question. In the past, when vaccinations enjoyed a status of a relatively low salience issue, there was not a large partisan gap on attitudes about childhood vaccinations about diseases like measles - majorities in both parties tended to support these positions, even though small gaps between Republicans and Democrats existed (Stecula, 2019). Partisanship also does not play a dominant role in determining support for childhood vaccine mandates, where majorities of both Democrats and Republicans tend to support such policies, pre-COVID (Stecula et al., 2020). Some even speculated that it was people on the left who were generally more "anti-vaccine," with the stereotype of "left-wing, coastal, white, wealthy moms" (Rogers, 2021).

In recent years, however, Republicans are more likely to endorse vaccine related misinformation (Motta, 2021) and are less likely to trust medical experts promoting vaccines (Stecula et al., 2020). This, coupled with the salience of COVID-vaccines relative to other vaccinations, as well as the politicization of COVID as a domain, renders it unsurprising that attitudes about COVID vaccines would follow the same politicized path as other COVID attitudes. Indeed, recent survey research documents large differences between Democrats and Republicans in willingness to obtain COVID vaccine, for example (Fridman et al., 2021; Callaghan et al., 2021).

This politicization and polarization of COVID-related attitudes and behaviors stems partly from the news media transmitting elite cues to the public. Public opinion, in general, 
tends to follow elites (Zaller, 1992; Lenz, 2012), and the dynamic of politicizing an issue in the news and elite cues shaping resulting opinion has been documented in other contexts (Merkley \& Stecula, 2021). This is potentially problematic, as coverage of COVID in right wing news outlets has tended to feature more misinformation (Motta et al., 2020; Evanega et al., 2020), more politicians and politicized statements (Sol Hart et al., 2020), and generally more antivaccine content (Savillo et al., 2021) than other sources. In short, Fox News, from the early days of the pandemic has engaged in spreading COVID and vaccine related misinformation, ranging from spreading COVID-related conspiracy theories, to casting doubt on COVID vaccine safety and effectiveness (McCann Ramirez, 2022). Taken together, this leads us to our first hypothesis. We expect news coverage on Fox News to be more negative in tone than news coverage of COVID vaccines in other mainstream news sources (Hypothesis 1).

We also expect that news content influences people's attitudes about COVID vaccinations. Our expectation is rooted in the fact that messaging needs to come from a trusted source to be effective, and Fox News remains the most trusted outlet among Americans on the political right (Jurkowitz et al., 2020). In fact, recent trends show that trust in the news has declined sharply, especially among conservatives, which further amplifies the importance of Fox News as a source of information on the right (Gottfried \& Liedke, 2021).

Our expectations, in this respect, are two-fold. First, we expect that increased negative coverage of COVID vaccinations on Fox News will result in an increased number of reports to VAERS - which we employ as a highly sensitive time-varying proxy for vaccine sentiment (Hypothesis 2a); i.e., because reports are aggregated at the daily level, as opposed to lessfrequent opinion survey research (see: Motta \& Stecula, 2021). Likewise, we expect that regular 
self-reported Fox News viewers in public opinion surveys will to exhibit higher levels of vaccine hesitancy (i.e., an unwillingness to vaccinate; Hypothesis $2 \mathrm{~b}$ ).

\section{Analytical Strategy}

Our analysis proceeds in three parts. First, we offer a descriptive assessment of Hypothesis 1 by comparing average daily fluctuations in the affective tone of COVID-19 vaccine media coverage across cable news outlets (Fox News, MSNBC, and CNN). We also include mainstream television news coverage in our analysis in order to draw comparisons to non-partisan media content across the televised medium ( $\mathrm{ABC}, \mathrm{CBS}$, and $\mathrm{NBC}$ News), as well as mainstream print news coverage (Associated Press, The New York Times, and The Washington Post) in order to draw comparisons between television and print mediums. If Hypothesis 1 is supported, Fox News should, on average, feature higher levels of negatively toned COVID-19 vaccine media coverage than all other outlets. Additional information about how we collect these data and operationalize media coverage tone can be found below (see: Data).

Additionally, we offer a more-rigorous statistical test of Hypothesls 1 by constructing an OLS model that regresses the tone of each COVID-19 story in our dataset on dichotomous indicators of whether that story originated from one of the three partisan cable news outlets, a mainstream television source, or a mainstream print source. We account for the possibility of correlated prediction errors over time, with respect to how media outlets discuss COVID-19 vaccine related issues, by clustering standard errors at the daily level.

Second, we test Hypothesis 2 by (1) plotting the relative daily number of reports submitted to VAERS (i.e., the total number of reports divided by the number of vaccines administered that day) - which, as we argued earlier, can serve as a proxy for shifts in public 
vaccine sentiment - as a function of (2) lagged average daily tone fluctuations across televised media. This allows us to descriptively assess whether or not change in the previous day's media coverage might then correspond to an uptick in side effect reports to VAERS.

We offer a more-formal statistical test of Hypothesis 2 by calculating the Pearson correlation coefficient between lagged coverage and side effect reports. As a robustness check, we also test for the possibility of reverse causation - i.e., that media outlets are responding to increased side effect reports by featuring more negative vaccine coverage - by assessing the correlation between lagged VAERS reports and daily media tone fluctuations.

Third, although we make an effort to validate VAERS as a proxy for public vaccine sentiment, we recognize that adverse event reports are imperfect proxy for public vaccine sentiment. Consequently, we fielded a series of six public opinion surveys over the first year of the COVID-19 pandemic that asked respondents to self-report their media viewing tendencies as well as their intentions to vaccinate against COVID-19. We use this data to construct a logistic regression model (with survey-level fixed effects) that regresses vaccine uptake intentions on self-reported exposure to each of the three cable news networks, mainstream television outlets, and mainstream print media. These models also account for other wellstudied correlates of intentions to vaccinate against COVID-19, including negative attitudes toward scientists and experts, gender (as women are more likely to intend to refuse COVID-19 vaccination), race (as non-White individuals are more likely to intend to refuse vaccination), and age (as younger Americans are more likely to intend to refuse vaccination). (See: Daoust, 2020; Callaghan et al., 2021; Merkley \& Loewen, 2021; Ruiz \& Bell, 2021). 
If Hypothesis 2 is supported, we would expect to observe higher levels of vaccine hesitancy among regular Fox viewers - i.e., because they are exposed to more negative content about the COVID-19 vaccine (as documented via our first two analytical tasks) - than those who do not regularly view Fox News.

\section{Data \& Measures}

Data for this study come from three sources: (1) both daily-aggregated and disaggregated media coverage of COVID-19 vaccine related issues, (2) adverse event reports from COVID-19 vaccination to VAERS, and (3) six original public opinion surveys that include measures of vaccine uptake intentions and self-reported media use. We describe each data source, as well as its corresponding measures, in turn.

\section{Media Content Data}

The news media data was collected from Lexis Nexis Academic. We searched for stories containing references to either "coronavirus" or "COVID" in addition to mentioning either "vaccine", "vaccination" or "vaccinated." We focused on the most important national news media outlets, including mainstream national print sources (The New York Times, The Washington Post, USA Today, as well as the Associated Press), traditional broadcast television ( $A B C$ News, NBC News, CBS News), as well as cable news outlets CNN, MSNBC, and Fox News. In total, we have obtained $\mathrm{N}=17,454$ articles and transcripts from these sources from December 1, 2020 until the end of April, 2021. Traditional print sources make up 53\% of the total news media sample, broadcast television transcripts make up $15 \%$ of the sample, CNN makes up $22 \%$, while Fox News and MSNBC are both each $5 \%$ of the sample. 
Our timeframe includes the period right before the emergency approval of the first COVID vaccines in the United States (Pfizer-BioNTech vaccine received the emergency use approval on December 11, 2020, while Moderna followed on December 18, 2020) and includes several months when vaccines were introduced to Americans and vaccination efforts ramped up, thus representing a period of time of utmost importance in terms of shaping public perceptions about COVID vaccines.

We measure sentiment of vaccine coverage using automated, dictionary based approaches. The unit of analysis is a story, and we employ the Linguistic Inquiry and Word Count (LIWC) software to compute tone, as measured by the ratio of negatively to positively valanced words in each story, standardized on a scale ranging from 0 (most negative) to 100 (most positive). LIWC has been extensively validated and used in a variety of different social scientific contexts (Tausczik \& Pennebaker, 2010; Motta \& Stecula, 2021). In general, dictionary methods have been shown to accurately capture basic sentiment of text, and these dictionaries have been successfully validated with human coders (Young \& Soroka, 2012).

\section{Vaccine Adverse Event Reporting System (VAERS) Data}

Next, we study daily fluctuations in the total number of adverse events reported to the VAERS database. VAERS is a service provided by the US Department of Health and Human Services that enables individuals either on their own and/or in consultation with their physicians to report adverse events after having received a vaccination. The event data used in this analysis were last updated on May 21, 2021. This cutoff date represents a period following the widespread availability of COVID-19 vaccines for nearly all Americans (thereby making it theoretically plausible to detect uptake effects across the general population), and prior to a plateau in 
vaccine uptake in the early summer months (which might complicate detecting variation in uptake intentions and/or adverse event reports).

Data from VAERS reports is unverified; meaning that there is not necessarily medical documentation that people actually suffered these side effects, or that vaccines were the cause. As we argue elsewhere (Motta \& Stecula, 2021) the unverified nature of VAERS reports are in some ways unideal for assessing the medical risks of vaccination. However, because nearly anyone can register a report with HHS, VAERs reports are ideal for our purposes; as they produce sensitive proxies of change in public sentiment toward vaccines.

We measure the Proportion of Daily Adverse Event Reports by summing up the total number of COVID-19 related reports $(\mathrm{N}=187,985)$ to HHS, aggregated at the daily level. We then calculate the relative proportion of reports submitted by dividing each daily aggregation by the total number of vaccines administered on that day, via data from Mathieu and colleagues (2021). We prefer to focus on the proportion (rather than the raw number) of reports (e.g., as Motta \& Stecula, 2021 do in their paper), due to the unprecedentedly swift nature of the COVID-19 vaccine rollout. In other words, because COVID-19 vaccine uptake increased dramatically over time, we would also expect the total number of VAERS reports submitted to be auto-correlated with time. Calculating fluctuations in side effect reports as a function of the number of vaccines administered daily allows us to remove that potential time confound from our analysis.

Additionally, we remove $\mathrm{N}=769$ reports that are improperly dated (i.e., alleging - due to result reporting error - the experience of symptoms months or years before January 1st 2020; weeks before the spread of COVID-19 to the US, and months before the development of 
vaccines). We then divide each daily aggregation by the total number of vaccines administered that day, based on data from the CDC (Mathieu et al., 2021). All of the data and syntax necessary to replicate the analyses presented in this paper is available at the Open Science Foundation at $<$ LINK $>$. Additional descriptive statistics about the VAERS data can be found in the Supplementary Materials.

\section{Public Opinion Data}

Finally, we fielded a six-wave, rolling cross-sectional public opinion survey throughout the first year of the COVID-19 pandemic that assessed public attitudes toward vaccination, self-reported media use, and a wide variety of other questions related to politics and public health. We fielded each wave bi-monthly from April 2020 to February 2021, and sampled approximately N $=1,000$ respondents (with the exception of the February 2021 wave, which included $N=1,501$ respondents). In total, the combined dataset had $\mathrm{N}=6,499$ responses.

We fielded each wave in partnership with Lucid Theorem, who used quota sampling procedures to ensure demographic representativeness with respect to respondents' age, race, gender, household income, political party, educational attainment, and Census region. To account for remaining deviations between the sample and population, we calculated a series of wave-specific survey weights that adjust respondents' age, gender, household income, race, and educational attainment to Census benchmarks. A comparison of weighted and unweighted data to known population benchmarks from each wave can be found in the Supplementary Materials.

The institutional review board at [university blinded for review] approved the data collection protocols employed in this study. Respondents provided documentation of informed 
consent prior to participating in the survey by affirming that they read a brief description of the study, and were compensated in exchange for their participation by Lucid Fulcrum, per the terms of their existing agreements with the company's opt-in panel service. Given the study's minimal time burden, the opt-in nature of the Lucid platform, low risk of adverse events, and the potential public health benefits of this research, we believe that monetary compensation in exchange for participation is well justified.

The primary outcome variable in our analyses is a dichotomous indicator of whether or not individuals intend to vaccinate against COVID-19. We measured COVID-19 Vaccine Hesitancy by asking respondents "When a vaccine for the novel coronavirus (COVID-19) becomes widely available, how likely are you to request to be vaccinated?" (see: Motta et al., 2021; Callaghan et al., 2021 for examples of studies using this approach to measuring COVID-19 vaccination intentions). Respondents could reply that they were "very likely," "somewhat likely," "not too likely," or "not likely at all" to do so. We re-scored this variable to take on a value of 0 if respondents definitely or probably planned to pursue vaccination - or, alternatively, if they reported having already been vaccinated on a separate question administered in the study's February 2021 wave - and 1 if they definitely or probably did not plan to vaccinate.

We also measured Self-Reported Media Use by asking respondents whether or not "in the past month" they had "watched or streamed" several different television programs. Respondents could report that they watched each program "almost every day," "about once a week," "just once or twice," or "never." We re-coded response options in proportion to their relative monthly viewership frequency, on a scale ranging from 0-1; i.e., people who watched 
each program daily received a score of 1 , whereas people who watch about once a week received a score of 0.17 (5/30; assuming a thirty day month with approximately five weeks), 0.07 if they watched only once or twice per month (2/30), or 0 if they reported never watching that program.

Because our survey asked respondents to report their media viewing habits across dozens of print, televised, and online sources, we were able to create media exposure analogues for each of the sources available in the media content data. We measure cable news viewership via questions probing self-reported exposure to programs on Fox News, CNN, and MSNBC. We also measure mainstream TV news exposure $(A B C, C B S$, and $N B C$ in the media content data) using a question which asked respondents whether they watch "national news broadcasts (e.g., $A B C, C B S, N B C)$ ". Finally, we measure print media consumption via an indicator of how frequently respondents reported reading the New York Times (one of the three print sources included in the media data, as no other print sources were available in the public opinion data).

Finally, our models control for a series of social and demographic factors, which, - as noted earlier - have been linked to COVID-19 vaccine refusal in past research. First, we include a standard measure of anti-intellectual attitude endorsement (developed by Oliver \& Rahn, 2016, and employed as an indicator of anti-intellectualism in several recent studies; e.g., Motta, 2018; Merkley, 2020), which asked respondents whether they would rather place their trust in the wisdom of "ordinary people" over "experts and intellectuals." We offered response options on a seven-point Likert scale; recoded to range from $0-1$, such that a score of 1 indicates strongly valuing the opinions of ordinary people over experts and intellectuals. 
We also control for respondents' age (binary indicators of whether respondents are aged $18-25 ; 25-44 ; 44-65 ; 65+$ ); gender (with self-identified women receiving a score of $1 ; 0$ otherwise); educational attainment (a binary indicator of whether or not respondents completed college); race and ethnicity (binary indicators of whether respondents identify as Black or Latinx); and survey wave (i.e., wave assignment indicators).

\section{Results}

We begin our analysis by investigating whether or not Fox News' coverage of COVID-19 vaccine related issues was more negatively toned than coverage from other programs $(\mathrm{H} 1)$. The results are presented in Figure 1.

As noted in our Analytical Strategy, the left-hand panel first offers a descriptive look at daily fluctuations in the average tone of COVID-19 across cable news networks (smoothed, via locally weighted regression). For within-medium comparisons, we also include mainstream TV news, and (for across-media comparisons) mainstream print news as well.

We find that, throughout the series, Fox News (bolded line) consistently featured morenegatively toned coverage than all other cable networks, as well as mainstream television news. At times, print media eclipses Fox with respect to the average negativity of its coverage. However, deviations from this general pattern are typically short-lived and infrequent.

The right-hand panel in Figure 1 offers a more formal summary of the differences across platforms by calculating predicted levels of negativity (linear predictions) via the multiple regression procedures described earlier. There, we again find that Fox News $(B=8.49, p<0.01)$ tends to feature significantly more negative coverage than all other outlets. Average daily tone is approximately 3 percentage points (on a scale ranging from 0-100) higher on Fox (44\%) than 
its next closest competitor (MSNBC; 41\%). As documented by the non-overlapping confidence intervals between the linear predictions presented in the Figure, this represents a statistically significant effect.

Having documented that coverage of COVID-19 related issues was more negative on Fox, we next consider the effects of changes in tone on adverse event reports to VAERS. Figure 2 plots average daily proportion of reports submitted to VAERS (in black) and lagged changes in tone (again, both smoothed via locally weighted regression).

Having documented that coverage of COVID-19 related issues was more negative on Fox, we next consider the effects of changes in tone on adverse event reports to VAERS. Figure 2 plots average daily proportion of reports submitted to VAERS (in black) and lagged changes in tone (again, both smoothed via locally weighted regression).

Before moving on, it is important to note that only a select group of frontline healthcare workers and highly vulnerable people were able to receive COVID-19 vaccines in the initial stages of the vaccine rollout. States also moved through the process of opening up vaccine administration to the general public at different speeds, and using a wide variety of selection criteria. It is consequently difficult to pinpoint a specific date at which vaccination started to become available for a broader swath of American adults (population to whom we wish to generalize). Although the selection of a date is necessarily arbitrary, we opted to begin our analysis on $2 / 1 / 2021$; i.e., on the first day following the first full month of vaccination.

Figure 1. A Comparison of Differences in the Tone of COVID-19 Coverage Across Media Platforms, \& Over Time 

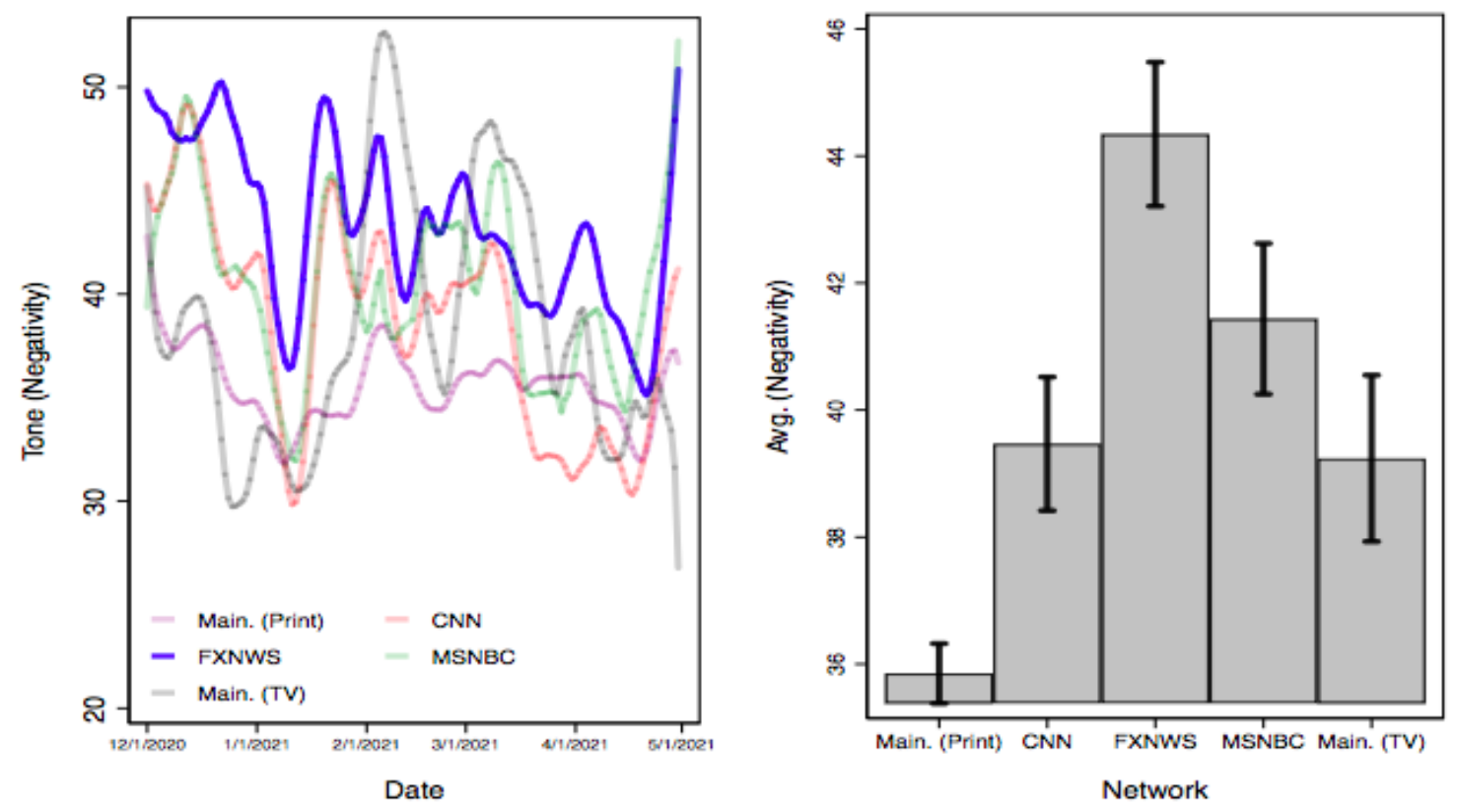

Note. The left-hand panel presents the aggregated daily average fluctuations in vaccine coverage tone across sources. For ease of visual interpretation, we present this quantity via locally weighted regression (bandwidth = 0.10) aggregated at the daily-level, across networks. The right-hand panel presents predicted levels of negativity (linear predictions) across cable news outlets throughout the duration of the series. Predictions are derived from an OLS model that regresses the disaggregated tone of each story $(N=17,454)$ on media source, with clustered standard errors at the daily level.

The results in Figure 2 offer strong support for our theoretical expectations. Descriptively, as evidenced by the comparatively smaller gaps between the locally-weighted regression lines throughout most of the series, the figure shows that the tone of Fox's media coverage moreclosely tracks with fluctuations in VAERS reports over time than that of other media networks.

The tone of CNN's vaccine coverage at times rivals that of Fox with respect to its consonance with VAERS reports, although the two trendlines diverge in mid-March; a period in which a comparatively more representative cross-section of the public had the opportunity to vaccinate (and, consequently, potentially submit reports to VAERS).

Figure 2. The Correspondence between Changes in COVID-19 Vaccine Coverage Tone and Reports to VAERS Across Media Platforms, \& Over Time 

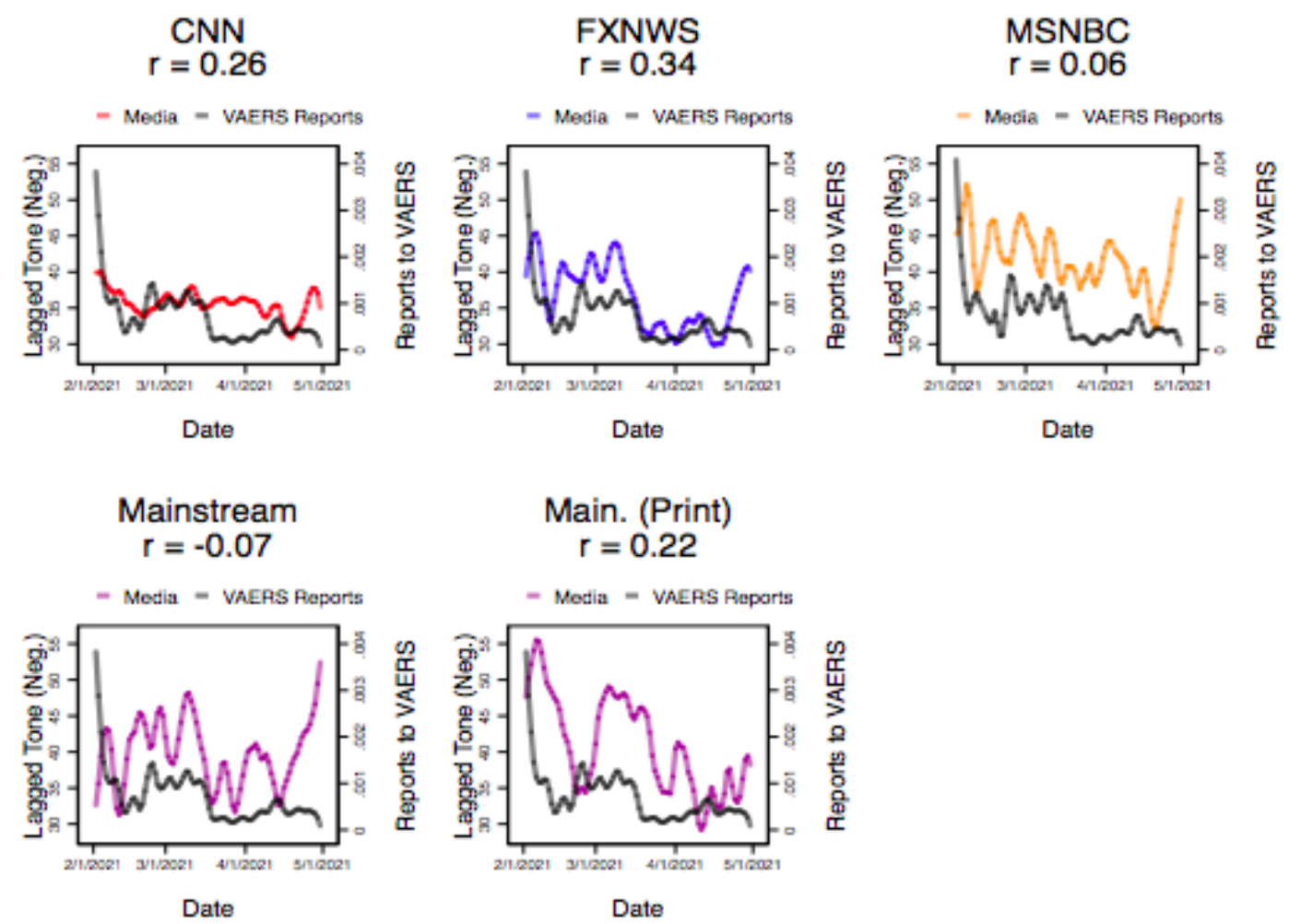

Note. Figure plots average daily tone fluctuations of vaccine-related media coverage across each televised sources, as well as average daily fluctuations in COVID-19 vaccine related reports to VAERS. For ease of visual interpretation, both quantities are presented using a locally weighted regression estimator (bandwidth $=0.10$ ). Correlation coefficients -- based on raw daily tone aggregations (as opposed to locally-weighted or "smoothed" estimates) -are provided on top of each panel. Note that, as we are primarily interested in drawing substantive comparisons across televised media sources, and because print media had significantly lower levels of negative tone throughout the series, we do not include a comparison to print media in this figure.

The results in Figure 2 offer strong support for our theoretical expectations. Descriptively, as evidenced by the comparatively smaller gaps between the locally-weighted regression lines throughout most of the series, the figure shows that the tone of Fox's media coverage moreclosely tracks with fluctuations in VAERS reports over time than that of other media networks. The tone of CNN's vaccine coverage at times rivals that of Fox with respect to its consonance with VAERS reports, although the two trendlines diverge in mid-March; a period in which a comparatively more representative cross-section of the public had the opportunity to vaccinate (and, consequently, potentially submit reports to VAERS). 
The figure also offers strong statistical support for our expectations. As the correlation coefficients printed above each panel suggest, the (lagged) tone of Fox's COVID-19 vaccine coverage is positively associated with increased adverse event reports over time. Although the tone of COVID-19 vaccine news coverage on CNN $(r=0.26, p<0.01$; two-tailed) and in print media $(r=0.22, p<0.01)$ is correlated with VAERS report frequency in excess of $r=0.20$, only one network - Fox News $(r=0.34, p<0.01)$ - boasts a correlation coefficient greater than 0.30 .

We recognize, of course, that VAERS is, at best, an imperfect proxy for public vaccine sentiment. We also recognize that correlational assessments of media influence may be spurious; i.e., influenced by other factors not observed in our data.

Consequently, Figure 3 presents the results of the multivariate micro-level survey analyses that assess the effects of media viewing habits on vaccine hesitancy. The figure plots change in vaccination uptake intentions (untransformed parameter estimates from a linear probability model) for daily cable news viewers across each media outlet, with mainstream television and print viewership included as controls.

We limit our analyses to self-identified Republicans, given the strong overlap between partisan identification and regular Fox News viewership (Stroud 2011). Because our opinion data are cross-sectional, we cannot disentangle the causal ordering of media consumption habits and partisan preferences, thereby posing endogeneity concerns. By subsetting our data in this way, we rule out the possibility that any media effects we might observe are instead the result of partisan differences (as partisanship is held constant).

Figure 3. The Effect of Fox News Viewership on COVID-19 Vaccination Intentions for SelfIdentified Republicans 


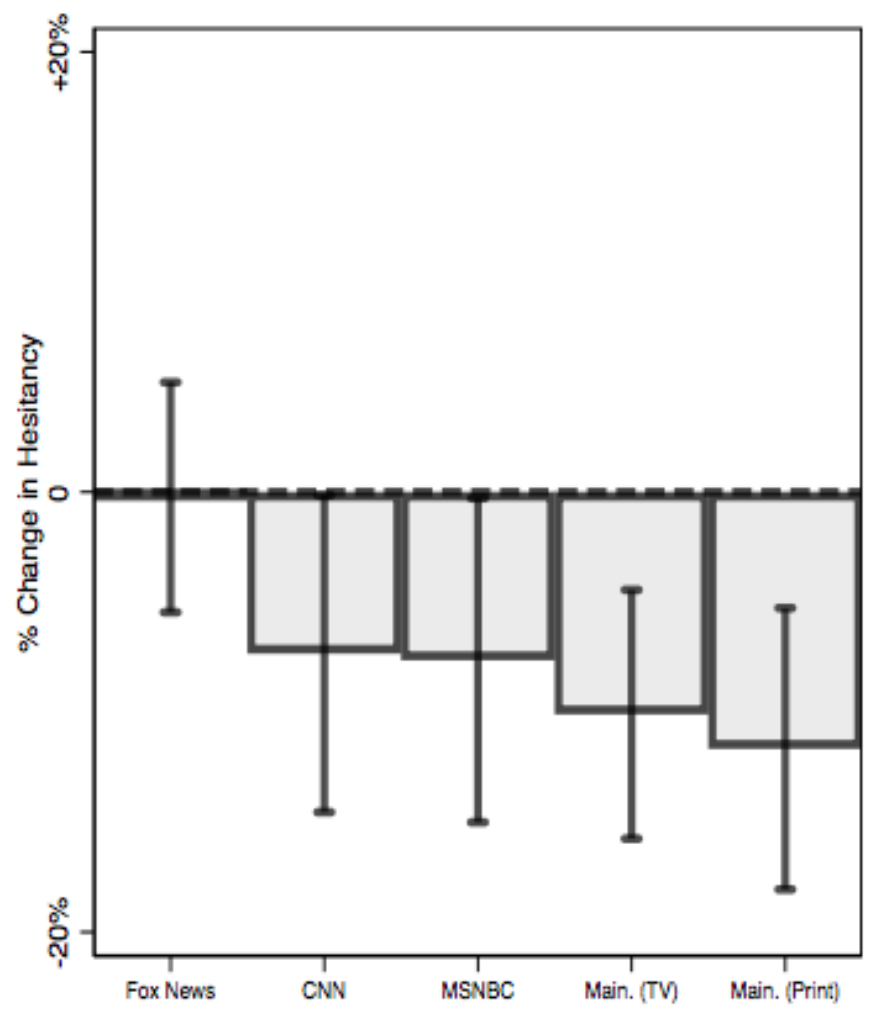

Note. Untransformed parameter estimates (\$|beta\$) from a linear probability model regressing the likelihood that survey respondents self-report intending to vaccinate against COVID-19 as a function of media viewing habits across televised and print platforms. Because all variables are scored to range from 0-1, parameter estimates can be interpreted as the percentage point change in vaccination intentions when comparing people who self-report never consuming information from each media source, compared to those who self-report doing so on a daily basis. Full model output is available in the Supplementary Materials.

The results presented in Figure 3 suggest that higher levels of exposure to every media outlet except for Fox News is negatively and significantly associated with vaccine refusal, among self-identified Republicans. For example, Republicans who regularly view mainstream television news are 10 percentage points less likely to report that they do not intend to vaccinate, compared to people who report never watching those programs.

Fox News, however, is the only exception to this pattern. Regular Fox viewers were neither more nor less likely than individuals who never view the program to intend to vaccinate against COVID-19 ( $\mathrm{B}=0.00, \mathrm{p}=0.93)$. Formal coefficient comparisons (Wald Tests) further 
suggest that the effects of Fox News viewership are statistically distinguishable from those of mainstream television viewership $(F=5.92, p=0.02)$ and print readership $(F=6.84, p=0.01)$ at conventional two-tailed levels of significance.

Overall, then, we find that Fox News consumption did not necessarily increase vaccine hesitancy among regular viewers. However, the null effect of Fox news viewership with respect to decreasing vaccine hesitancy stands in sharp contrast to the ameliorative effects of consuming any other news media outlet.

\section{Robustness Checks \& Sensitivity Analyses}

Finally, before concluding, we consider several conceptual and empirical objections that some might have to the analyses presented so far. We describe each potential concern, and how we assess each one, in turn.

Sentiment vs. Substance

At this point, some might object that the differences in tone that we observe across partisan cable news outlets - and their differential effects on adverse event reports - does not necessarily shed light on differences in substance across platforms. For example, careful inspection of Figure 1 reveals that MSNBC - which is typically preferred by left-leaning news audiences (Stroud 2011), and may (due to the partisan dynamics of COVID vaccine uptake and promotion; Callaghan et al., 2021; Gadarian et al., 2021) be more amenable to talking about COVID-19 vaccination in a positive light - features the second most-negative COVID vaccine coverage.

MSNBC's negativity could result from several factors, including the inherent methodological limitations of assessing negative tone from dictionary-based text analysis 
approaches and/or differences in the substantive context of negativity across platforms. This second concern is particularly important to take into account, as it could help explain why the correlation between negativity and adverse event reports is comparatively lower on MSNBC (vs. FXNWS), despite expressing similar levels of negativity overall. It could be the case, for example, that whereas negativity on Fox News primarily pertains to concerns about the safety, efficacy, and necessity of vaccinating against COVID-19, negativity on MSNBC is directed toward frustration with unequal access to vaccination, public vaccine skepticism, or the former President's reluctance to vaccinate in public.

If our theoretical expectations are correct, negative vaccine coverage on Fox should be particularly likely (vs. negative coverage on other outlets) to cast doubt on the benefits or to emphasize the risks of vaccination. To test this possibility, we built a series of logistic regression model wherein we (1) regress general adverse event keywords (i.e., "side effects"), as well as a series of specific side-effect concerns (i.e., "headache," "pain," "fatigue," "chills") used to refine our media sample search parameters (see: Methods), on news coverage tone, an indicator of the network responsible for airing that story, and the interaction between the two, and (2) subset the data to focus on those stories that are at least somewhat negatively valenced in tone (i.e., with a score in excess of $50 \%$ ). A positive and statistically significant interaction effect for negativity on FXNWS would indicate that FXNWS is more likely than other outlets to reference issues related to vaccine safety in a more-negative context.

We summarize the results in Figure 4, which plots the predicted probability that each story in our sample references the general "side effects" keyword across outlets, and over story-level negativity. Grayed lines represent predictions from interactive parameter estimates 
that fail to attain conventional levels of two-tailed significance, whereas black lines indicate those that do. Full model output, including analyses where we swap the dependent variable for specific side effect search terms, are included in the Supplemental Materials.

Figure 4. The Effect of Negativity \& Program on COVID-19 Vaccination Side Effect Mentions

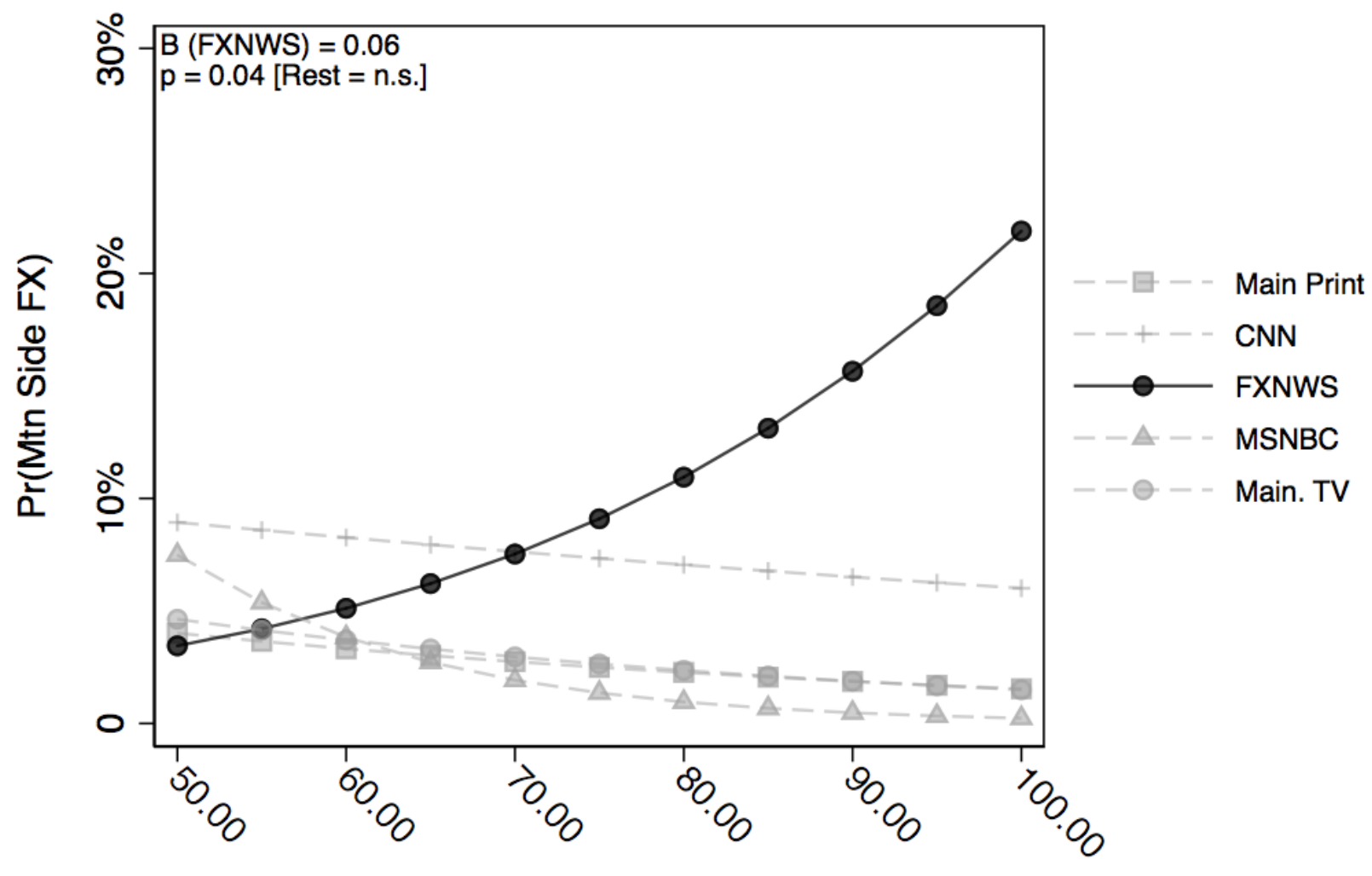

Negativity

Note. Predicted Probabilities from a logistic regression model presented. Model regresses a dichotomous indicator of whether or not stories included the term "Side Effects" on media outlet, story-level negativity, and the interaction between the two. Data are subset to include negative stories only (i.e., where tone is in excess of $50 \%$; $N$ $=4,101$. See Methods for additional information about the LWIC procedure for assessing tone). Full model output is available in the Supplementary Materials (Table S5). Note that confidence intervals are omitted for ease of visual interpretation (i.e., because only one interactive parameter estimate produced an effect that was statistically discernible from zero).

The results presented in Figure 4 suggest that elevated levels of negativity are associated with an increased likelihood of mentioning side effects (at the story level) for just one network; Fox News $(B=0.06, p<0.05)$. The likelihood that FXNWS stories mention vaccine 
side effects increases from $3 \%$ at low levels of negativity to $22 \%$ at high levels (a 19 percentage point increase). Although this analysis cannot capture the many ways in which the content of negative stories differs across networks, it nevertheless suggests that there are important substantive differences in negativity on FXNWS that can, theoretically, help explain why its coverage is associated with vaccine skepticism. Fox, compared to other networks, was more likely to talk about issues related to vaccine safety in its negative coverage, which was in turn associated with an increased likelihood to report side effects to VAERS.

Still, some might further ask why we choose to focus on differences in tone when studying adverse event reports, as opposed to mentions of side effects themselves. Our rationale for focusing on tone is, at least in part, due to data limitations. References to side effects (writ large) comprise just under $5 \%$ of all stories aired during the series, and references to specific side effects never vary widely in their incidence; e.g., whereas $12 \%$ of stories mention "pain," just 1\% mention "chills." Given that differences in tone across networks (Figure 4) are in part reflective of differences in side effect coverage in anticipated ways across networks, and recognizing that our side effect search terms reflect an imperfect attempt to measure the many ways in which the media can talk about the potential risks of vaccinating, we opt to treat tone as a "catch all" proxy for these concerns.

\section{Reverse Causality Assessment}

Furthermore, some might raise the possibility that the pattern of effects documented in Figure 2 may be reverse causal. Media outlets like Fox, for example, may feature more negative stories about the COVID-19 vaccine because they are reacting to changes public concern (as opposed to influencing it); as proxied by side effect reports. 
We test this possibility by calculating the correlation between lagged VAERS reports (again, using a one-day lag), and current media tone. We find that the correlation between these two quantities $(r=0.08)$ is substantively weaker than the quantities presented in Figure 2, and - unlike the correlated presented in Figure 2 - fails to attain statistical significance $(p=$ 0.44). Consequently, we think it is unlikely that changes in VAERS reports are responsible for driving Fox's comparatively more-negative coverage of the vaccine, and think it is instead more plausible that negative media coverage exerts a stronger influence on public vaccine sentiment.

\section{A Multivariate Analysis of for The Effects of Vaccine News Media Frequency}

Moreover, some might express concern that the effects documented in Figure 2 may not hold in multivariate models that account for the total number of COVID-19 vaccine-related stories aired on a particular date, on each network. Some might argue that the total volume of news stories produced about COVID-19 vaccines may raise the salience of vaccine-related issues, and thereby increase the extent to which Americans are concerned about potential side effects. Others might also express concern that periods in which the volume of COVID-19 vaccinerelated media coverage is higher also tends to be more negative in tone; i.e., because the media are responding to reports of adverse medical events, logistical hurdles with vaccine rollout, and other medical/logistical issues.

Although we lack a priori expectations about the effect of volume on vaccine hesitancy, and note that increased attention to vaccine-related issues may also be associated with positive vaccine developments (e.g., high public demand for vaccines in the early phases of vaccine rollout, reports of vaccine safety and effectiveness), we nevertheless account for this possibility in the Supplementary Materials. There, we present a series of multivariate OLS models that 
regress reports to VAERS (see: Figure 2) on lagged tone, as well as an indicator of the total number of COVID-19 related stories produced on each day represented in the series, on each network. To avoid excessive collinearity - as news outlets may produce a greater volume of stories on the same day, in response to vaccine-related events - we estimate these effects in separate models for each media outlet studied in Figure 2.

In Table S4, we again find strong evidence in favor of the pattern of results presented in Figure 2. Even when accounting for change in the lagged frequency of COVID-19 vaccine media coverage, we find that the effect of negative COVID-19 vaccine coverage on Fox is both positively $(B=0.003)$ and significantly $(p<0.05)$ associated with increased reports to VAERS. No other network's coverage exhibits a statistically significant relationship with adverse event reports. We also fail to detect an effect of the volume of COVID-19 vaccine coverage on adverse event reports.

Convergent Validity Assessment: VAERS as a Proxy for Public Opinion

Additionally, we recognize that VAERS reports are an imperfect proxy for public opinion concerning COVID-19 vaccine hesitancy. In addition to the micro-level validation analyses presented in Figure 3, we also consider the extent to which VAERS reports are correlated with vaccine uptake opinion data.

This, of course, is a challenging exercise. While many survey research outlets have attempted to measure vaccine hesitancy throughout the COVID-19 pandemic, few have attempted to do so regularly, using the same sampling methods and uptake questions. Moreover, given the limited window of time in which the COVID-19 vaccine has been available, 
even weekly tracking polls offer us few opportunities to assess the correspondence between the two data sources.

Nevertheless, as a supplement to Figure 3, we offer a supplementary Figure (Figure S2) that assesses the correspondence between the weekly average number of respondents who report intending to vaccinate (or who have already vaccinated) in YouGov/The Economist's weekly COVID-19 tracking poll. Although we have just over four months' worth of tracking poll data available to study ( $N=18$ weekly estimates), we document a strong negative correlation ( $r$ $=-0.74)$ between vaccine uptake - as measured in the polling data - and side effect reports to VAERS. This analysis, while correlational and highly preliminary, offers additional evidence in favor of using VAERS reports as a proxy for public vaccine sentiment.

\section{Sensitivity Analyses}

Finally, we test whether the results obtained from our multivariate models are sensitive to the estimation strategies we employ. For example, we recognize that some might prefer that we account for the possibility of correlated prediction errors when testing for differences in tone content across cable news and other media via hierarchical linear modeling (as opposed to the implementation of clustered standard errors). We include these results in Table S2 (where we also present the original results used to calculate the linear predictions in Figure 1). There, we detect an analogous pattern of effects across estimation strategies.

Likewise, we recognize that some might object to the use of OLS regression when modeling vaccine hesitancy in the public opinion data (which is measured dichotomously). Consequently, we re-estimate the models presented in Figure 3 using logistic regression. The results, are presented in Table S3 (which, again, includes the full model output used to generate 
the results presented in Figure 3). There, we again recover a pattern of effects analogous to those used to produce the findings presented in Figure 3.

\section{Discussion and Conclusion}

In this paper, we provide novel, multi-method evidence that media coverage of COVID vaccines has been markedly different on Fox News compared to other mainstream, national news outlets. We also demonstrate that differences in coverage influences public vaccine attitudes and behavior, as expressed through the analysis of over 180,000 vaccine adverse event reports to the Department of Health and Human Services, as well as six original surveys of over 6,000 adult Americans. Specifically, our work finds that increases in negative COVID-19 vaccine coverage on Fox News is associated with vaccine skepticism at the macro level (VAERS data), and that increased Fox news viewership is associated with intentions to refuse vaccination at the micro level (opinion data).

Our work demonstrates the usage of the VAERS database as an effective proxy for public vaccine sentiment, which more researchers should be using to understand how Americans feel about vaccinations. In the context of the pandemic, the anti-vaccine groups and political actors on the conservative right have been using VAERS to sow doubt and distrust about COVID-19 vaccinations. ${ }^{4}$ That has been an unfortunate development, given the general lack of understanding of VAERS among the general public. But the positive aspect of the growth of VAERS is the ability of this dataset to inform researchers of how American vaccine skepticism changes over time.

\footnotetext{
${ }^{4}$ https://theconversation.com/unverified-reports-of-vaccine-side-effects-in-vaers-arent-the-smoking-guns-portrayed-by-rightwing-media-outlets-they-can-offer-insight-into-vaccine-hesitancy-166401
} 
Finally, we exhibit the persuasive power of partisan news media in the United States, even amid the unprecedented challenges of a global pandemic. While some might have expected the promise of ending a global pandemic to interrupt conventional media effect processes, we find that differences in covered vaccine-related issues had both predictable and polarizing effects on public opinion.

Many of the prime time hosts on Fox News, such as Tucker Carlson or Sean Hannity, have been casting doubt on COVID vaccines ${ }^{5}$ and disparaging mask wearing ${ }^{6}$ during the pandemic, despite themselves being subjected to strict COVID policies. Yet, they frequently shirked responsibility for the content they were producing. Hannity has previously declared that he is not a journalist, ${ }^{7}$ while Carlson has been presented by Fox News' lawyers as someone who reasonable viewers cannot believe, because he is not stating actual facts but instead is engaging in exaggeration. ${ }^{8}$ But our work shows that regardless of how these commentators and their employer view themselves, the reality is that their audiences heed their advice. And when it happens during a global pandemic, the consequences of their actions are severe and affect not only their viewers, but also broader society.

\section{Bibliography}

Allcott, Hunt, Levi Boxell, Jacob Conway, Matthew Gentzkow, Michael Thaler, \& David Yang. 2020. Polarization and public health: Partisan differences in social distancing during the coronavirus pandemic. Journal of Public Economics, 191, 104254. https://doi.org/10.1016/j.jpubeco.2020.104254

Callaghan, Timothy., Ali Moghtaderi, Jennifer A. Lueck, Peter Hotez, Uli Strych, Avi Dor, Erika Franklin Fowler, \& Matt Motta. 2021. Correlates and disparities of intention to vaccinate against COVID-19. Social Science \& Medicine (1982), 272, 113638. https://doi.org/10.1016/j.socscimed.2020.113638

\footnotetext{
${ }^{5}$ https://www.nytimes.com/2021/07/11/business/media/vaccines-fox-news-hosts.html

${ }^{6}$ https://www.cnn.com/videos/business/2021/08/22/darcy-fox-news-host-spouts-anti-mask-venom-while-his-staff-wearsmasks.cnn

${ }^{7}$ https://www.washingtonpost.com/lifestyle/style/is-sean-hannity-a-journalist-or-not-heres-why-itmatters/2018/04/18/ca6b6bc2-431e-11e8-8569-26fda6b404c7_story.html

${ }^{8}$ https://www.npr.org/2020/09/29/917747123/you-literally-cant-believe-the-facts-tucker-carlson-tells-you-so-say-fox-s-lawye
} 
Clinton, Joshua., Jon Cohen, John Lapinski, \& Marc Trussler. 2021. Partisan pandemic: How partisanship and public health concerns affect individuals' social mobility during COVID-19. Science Advances, 7(2), eabd7204. https://doi.org/10.1126/sciadv.abd7204

Daoust, J.-F. 2020. Elderly people and responses to COVID-19 in 27 Countries. PLOS ONE, 15(7), e0235590. https://doi.org/10.1371/journal.pone.0235590

Evanega, Sarah, Mark Lynas, Jordan Adams, \& Karinne Smolenyak. 2020. Coronavirus misinformation: Quantifying sources and themes in the COVID-19 'infodemic.' Cornell Alliance for Science. https://allianceforscience.cornell.edu/wp-content/uploads/2020/09/Evanega-et-al-CoronavirusmisinformationFINAL.pdf

Fridman, Ariel, Rachel Gershon, \& Ayelet Gneezy. 2021. COVID-19 and vaccine hesitancy: A longitudinal study. PLOS ONE, 16(4), e0250123. https://doi.org/10.1371/journal.pone.0250123

Gadarian, Shana. K., Sara W. Goodman, \& Thomas B. Pepinsky. 2021. Partisanship, health behavior, and policy attitudes in the early stages of the COVID-19 pandemic. PLOS ONE, 16(4), e0249596. https://doi.org/10.1371/journal.pone.0249596

Gottfried, Jeffrey., \& Jacob Liedke. 2021. Partisan divides in media trust widen, driven by a decline among Republicans. Pew Research Center. Retrieved September 13, 2021, from https://www.pewresearch.org/facttank/2021/08/30/partisan-divides-in-media-trust-widen-driven-by-a-decline-among-republicans/

Green, Jon, Jared Edgerton, Daniel Naftel, Kelsey Shoub, \& Skyler J. Cranmer. 2020. Elusive consensus: Polarization in elite communication on the COVID-19 pandemic. Science Advances, 6(28), eabc2717. https://doi.org/10.1126/sciadv.abc2717

Grossman, Guy., Soojong Kim, Jonah M. Rexer, \& Harsha Thirumurthy. 2020. Political partisanship influences behavioral responses to governors' recommendations for COVID-19 prevention in the United States. Proceedings of the National Academy of Sciences, 117(39), 24144-24153.

Haglin, Kathryn., Daniel Chapman, Matt Motta, \& Dan Kahan. 2020. How localized outbreaks and changes in media coverage affect zika attitudes in national and local contexts. Health communication, 35(13), 1686-1697.

Hart, P. Sol. Sedona Chinn, \& Stuart Soroka. 2020. Politicization and Polarization in COVID-19 News Coverage. Science Communication, 42(5), 679-697. https://doi.org/10.1177/1075547020950735

Jurkowitz, Mark., Amy Mitchell, Elisa Shearer, \& Mason Walker. 2020, January 24. U.S. Media Polarization and the 2020 Election: A Nation Divided. Pew Research Center's Journalism Project. https://www.pewresearch.org/journalism/2020/01/24/u-s-media-polarization-and-the-2020-election-anation-divided/

Lecheler, Sophie., Claes de Vreese, \& Rune Slothuus. 2009. Issue importance as a moderator of framing effects. Communication research, 36(3), 400-425.

Lenz, Gabriel. S. 2012. Follow the Leader?: How Voters Respond to Politicians' Policies and Performance. University of Chicago Press.

McCann Ramirez, Nikki. 2022. "Timeline: Tucker Carlson's Unhinged COVID-19 Coverage." Media Matters for America. January 25, 2022. https://www.mediamatters.org/tucker-carlson/timeline-tucker-carlsons-unhingedcovid-19-coverage.

Mathieu, Edouard., Hannah Ritchie, Esteban Ortiz-Ospina, Max Roser, Joe Hasell, Cameron Appel, Charlie Giattino, \& Lucas Rodés-Guirao. 2021. A global database of COVID-19 vaccinations. Nature Human Behaviour, 5(7), 947953. https://doi.org/10.1038/s41562-021-01122-8

McCombs, Maxwell. 2005. A look at agenda-setting: Past, present and future. Journalism studies, 6(4), 543-557.

McCombs, Maxwell. E., \& Shaw, D. L. 1972. The Agenda-Setting Function of Mass Media. Public Opinion Quarterly, 36(2), 176-187. https://doi.org/10.1086/267990

Merkley, Eric. 2020. Anti-Intellectualism, Populism, and Motivated Resistance to expert consensus. Public Opinion Quarterly. https://doi.org/10.1093/poq/nfz053

Merkley, Eric, Aengus Bridgman, Peter J. Loewen, Taylor Owen, Derek Ruths, \& Oleg Zhilin. 2020. A Rare Moment of Cross-Partisan Consensus: Elite and Public Response to the COVID-19 Pandemic in Canada. Canadian Journal of Political Science/Revue Canadienne de Science Politique, 53(2), 311-318. https://doi.org/10.1017/S0008423920000311

Merkley, Eric., \& Peter J. Loewen. 2021. Anti-intellectualism and the mass public's response to the COVID-19 pandemic. Nature Human Behaviour, 5(6), 706-715. https://doi.org/10.1038/s41562-021-01112-w 
Merkley, Eric, \& Dominik A. Stecula. 2021. Party Cues in the News: Democratic Elites, Republican Backlash, and the Dynamics of Climate Skepticism. British Journal of Political Science, 51(4), 1439-1456. https://doi.org/10.1017/S0007123420000113

Motta, Matt 2018. The Dynamics and Political Implications of Anti-Intellectualism in the United States. American Politics Research, 46(3), 465-498. https://doi.org/10.1177/1532673X17719507

Motta, Matt 2021. Republicans, Not Democrats, Are More Likely to Endorse Anti-Vaccine Misinformation. American Politics Research, 49(5), 428-438. https://doi.org/10.1177/1532673X211022639

Motta, Matt., Dominik A. Stecula, \& Christina Farhart. 2020. How Right-Leaning Media Coverage of COVID-19 Facilitated the Spread of Misinformation in the Early Stages of the Pandemic in the U.S. Canadian Journal of Political Science/Revue Canadienne de Science Politique, 1-9. https://doi.org/10.1017/S0008423920000396

Oliver, J. Eric, \& Wendy M. Rahn. 2016. Rise of the Trumpenvolk: Populism in the 2016 Election. The ANNALS of the American Academy of Political and Social Science, 667(1), 189-206. https://doi.org/10.1177/0002716216662639

Pickup, Mark, Dominik A. Stecula, \& Clifton van der Linden, C. 2020. Novel Coronavirus, Old Partisanship: COVID-19 Attitudes and Behaviours in the United States and Canada. Canadian Journal of Political Science/Revue Canadienne de Science Politique, 53(2), 357-364. https://doi.org/10.1017/S0008423920000463

Pickup, Mark., Dominik A. Stecula, \& Clifton van der Linden, C.. 2021. American Exceptionalism: Determinants of spreading COVID-19 misinformation online in five countries. SocArXiv. https://doi.org/10.31235/osf.io/z547j

Rogers, Kaleigh. 2021, September 9. Republicans Aren't New To The Anti-Vaxx Movement. FiveThirtyEight. https://fivethirtyeight.com/features/republicans-arent-new-to-the-anti-vaxx-movement/

Ruiz, Jeanette. B., \& Robert A. Bell. 2021. Predictors of intention to vaccinate against COVID-19: Results of a nationwide survey. Vaccine, 39(7), 1080-1086. https://doi.org/10.1016/j.vaccine.2021.01.010

Savillo, Rob \& Tyler Monroe. 2021, August 19. Fox's effort to undermine vaccines has only worsened. Media Matters for America. https://www.mediamatters.org/fox-news/foxs-effort-undermine-vaccines-has-onlyworsened

Scheufele, Dietram. A., \& David Tewksbury. 2007. Framing, Agenda Setting, and Priming: The Evolution of Three Media Effects Models. Journal of Communication, 57(1), 9-20. https://doi.org/10.1111/j.14602466.2006.00326_5.x

Shafi, Ashik. 2017. Personal experience versus media coverage: Testing the issue obtrusiveness condition of agenda-setting theory in a developing country. Journalism \& Mass Communication Quarterly, 94(4), 10561072.

Stecula, Dominik. A. 2019, July 10. Vaccines should not become a partisan issue. Philadelphia Inquirer. https://www.inquirer.com/opinion/commentary/vaccines-outbreaks-exemption-bills-democrats-republicans20190710.html

Stecula, Dominik. A., Ozan Kuru, Dolores Albarracin, \& Kathleen H. Jamieson. 2020. Policy Views and Negative Beliefs About Vaccines in the United States, 2019. American Journal of Public Health, 110(10), 1561-1563. https://doi.org/10.2105/AJPH.2020.305828

Stecula, Dominik A., Ozan Kuru, \& Kathleen H. Jamieson. 2020. How trust in experts and media use affect acceptance of common anti-vaccination claims. The Harvard Kennedy School (HKS) Misinformation Review, 1(1). https://doi.org/10.37016/mr-2020-007

Stroud, Natalie. J. 2011. Niche News: The Politics of News Choice. Oxford University Press.

Tausczik, Yia. R., \& James W. Pennebaker. (2010). The Psychological Meaning of Words: LIWC and Computerized Text Analysis Methods. Journal of Language and Social Psychology, 29(1), 24-54. https://doi.org/10.1177/0261927X09351676

Yagade, Aileen., \& David M. Dozier. 1990. The media agenda-setting effect of concrete versus abstract issues. Journalism Quarterly, 67(1), 3-10.

Young, Lori, \& Stuart Soroka. 2012. Affective News: The Automated Coding of Sentiment in Political Texts. Political Communication, 29(2), 205-231. https://doi.org/10.1080/10584609.2012.671234

Zaller, John. 1992. The Nature and Origins of Mass Opinion. Cambridge University Press. 